Oliver Friggieri

dell'Università di Malta

\title{
INFLUSSI MANZONIANI SUL POETA NAZIONALE DI MALTA, DUN KARM
}

Saggio vincitore del

XIV Premio Letterario Silarus 1982

Estratto dalla rivista

SILARVS

PJ

9698.3

.P76
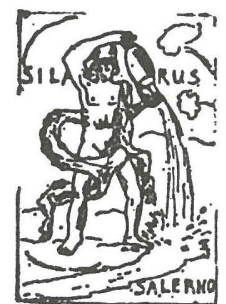

Z67

PB 203

$\mathrm{F}$ 


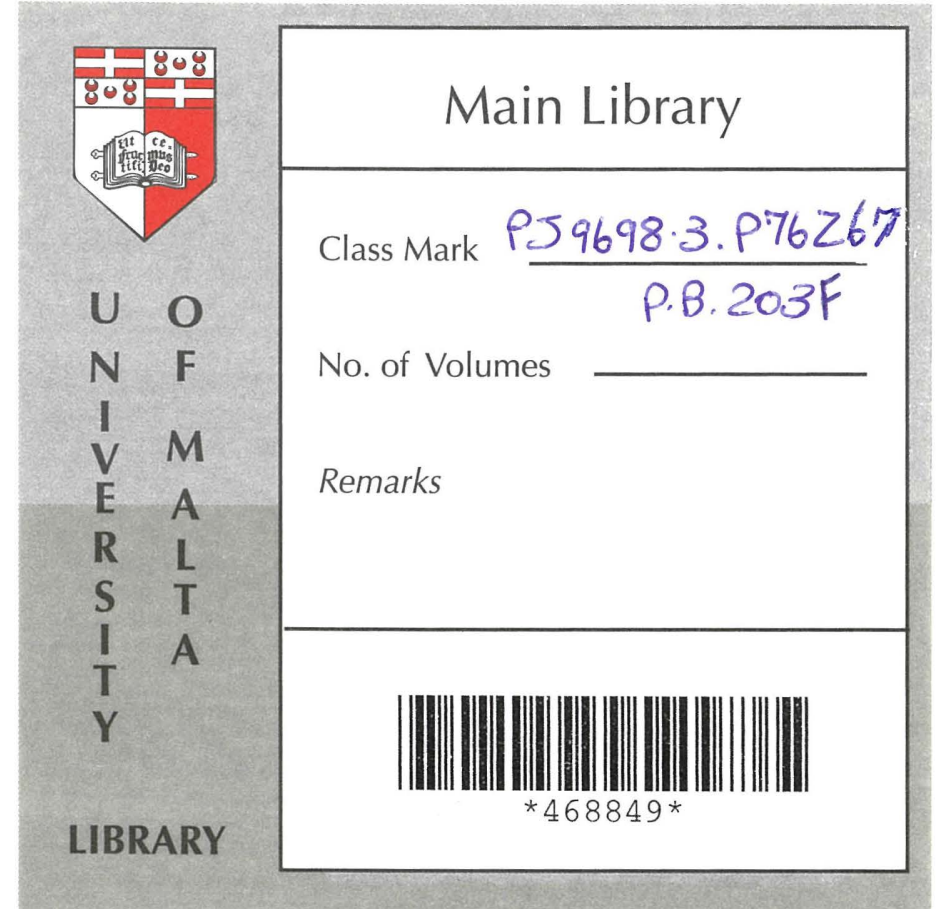




\title{
Oliver Friggieri
}

dell'Università di Malta

\section{INFLUSSI MANZONIANI \\ SUL POETA NAZIONALE DI MALTA, DUN KARM}

\author{
Saggio vincitore del \\ XIV Premio Letterario Silarus 1982
}

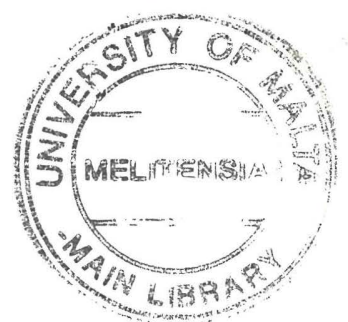

Estratto dalla rivista

SILARVS

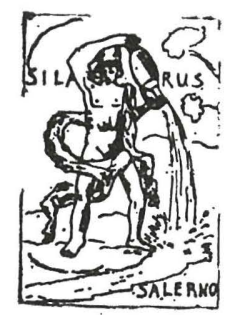




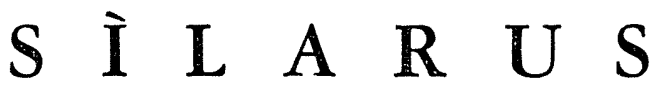

RASSEGNA BIMESTRALE DI CULTURA

Direttore: ITALO ROCCO

Redattore Capo: DOMENICO CICCARELLI

Direzione,

Redazione, Amministrazione:

84091 BATTIPAGLIA (Salerno)

Via B. Buozzi, 47 


\section{Oliver Friggieri \\ INFLUSSI MANZONIANI \\ SUL POETA NAZIONALE DI MALTA, DUN KARM}

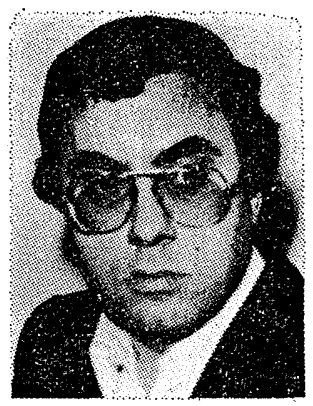

Nato a Malta nel 1947, Oliver Friggieri ha studiato presso l'Università di Malta e presso l'Università Cattolica di Milano (1977) dove ha fatto ricerca per la sua tesi del dottorato sull'influenza della letteratura italiana sulla poesia maltese. Autore di numerosi libri pubblicati sia a Malta sia all'estero. Membro dell' « Association Internationale Des Critiques Littéraires » (Parigi), e docente di Letteratura Maltese all'Università di Malta, egli è autore di numerosi saggi pubblicati su diverse riviste maltesi e straniere, e.g. in Italia, Siria, Scozia, America, Libia, Inghilterra, Grecia. I suoi libri: saggistica, narrativa, poesia, traduzione. Considerato il critico letterario più importante di Malta, O. Friggieri è autore, tra l'altro, della unica Storia della Letteratura Maltese che esiste. Ha anche raccolto, in una edizione critica completa (l'unica esistente) tutte le poesie del poeta nazionale di Malta, Dun Karm.

Il saggio cerca di determinare $i$ limiti $e$ il carattere della presenza del Manzoni nel poeta nazionale di Malta, Dun Karm (1871-1961). Il lavoro, condotto in termini comparatistici, analizza il lessico piu tipico del Manzoni e di Dun Karm, mette in rilievo $i$ riscontri, le assimilazioni e le remote risonanze. Alcuni degli elementi più significativi con cui si presenta questo confronto tematico-ideale tra $i$ due poeti sono le antonomasie, $i$ vocaboli, le metafore, le strofe.

Con questa ricerca esce fuori la figura di un Dun Karm manzoniano in alcuni momenti, e sempre pronto a riaffermare la propria identità di poeta maltese ma alla luce della cultura continentale, che per lui e per tanti altri autori dell'isola si fa particolarmente viva tramite la cultura italiana.

Gli influssi manzoniani si trovano sia nelle poesie italiane sia nelle poesie maltesi di Dun Karm.

Il poeta nazionale di Malta, Dun Karm Psaila (1871-1961), noto popolarmente come Dun Karm, è la voce più significátiva di tutta la letteratura tradizionale di Malta. In lui trovano eco tanti poeti italiani dell'Ottocento, particolarmente il Manzoni degli Inni Sacri, visto come lo spirito equilibrato che sintetizza una intera civiltà e che fonde in un unico insieme il passato e il presente.

L'influsso manzoniano è particolarmente suggerito dall'uso di forme poetiche identiche, ma ovviamente va oltre le condizioni formali. Un esempio fondamentale si trova nel caso della strofa pindarica, che consta di quattro, sei o più versi comunemente settenari, sdruccioli, piani e tronchi, che possono alternarsi variamente, a volontà del poeta. Tradizionalmente veniva adoperata in poesie di motivo religioso e patriottico, e suggerisce di poter essere cantata da un coro. Effettivamente il componimento prenderebbe il carattere e il nome di inno.

Nel caso specifico della letteratura italiana, l'uso della strofa faceva tornare nella poesia gli schemi pindarici, gli elogi sovrabbondanti, le sentenze che sottolineano, le esortazioni commosse e i rapidissimi passaggi. Gli Arcadi, influenzati dal Metastasio, dal Rolli e dallo Zappi di 
Imola, le aggiunsero una squisita fluidità musicale e le dolci e tenuamente malinconiche cadenze che si sciolgono facilmente in un motivo sonoro. Il Parini, come personaggio che incorpora in sè il modello dell'uomo moralmente equilibrato del tempo moderno, la tradusse in uno strumento di educazione etica e civile. Dopo di lui, la strofa subì diversi sviluppi sotto le mani del Monti, del Foscolo, del Manzoni, dello Zanella e del Carducci, tutte voci che nel suo eclettismo culturale Dun Karm conosceva e riecheggiava.

Dun Karm si serve di questo modello per una vastissima gamma di motivi, sia in italiano sia in maltese. La trama degli argomenti si inquadra essenzialmente entro la cornice della religione cattolica e dei misteri principali (1), ma include anche motivi di celebrazione augurale (2), allacciandosi naturalmente alla ricca produzione di poesie festose di cui è piena la letteratura italiana, specialmente quella del Settecento. Esempi anche più prossimi aveva Dun Karm negli echi maltesi di quella produzione innografica, dal Ciantar al Testaferrata al Caruana.

In conseguenza al fatto che il poeta segue il felice connubio di nucleo tematico e di componenti ritmiche che figura in poeti italiani, quali sono il Monti e il Manzoni, cerca pure di creare un parallelismo tecnico quasi assolutamente identico. Ad esempio, l'inno pindarico $L a$ Risurrezione del Manzoni è costruito in sedici strofe di sette versi ottonari ciascuna. Ogni coppia di strofe è musicalmente legata con la seguente dalla rima comune del verso finale, che è sempre tronco: suscitò - risveglio; ristè - te; Redentor -Vincitor; giurò - ricordò; tramortì qui; annunziar - pregar; vestir-apparir; verrà-risorgerà. Gli altri versi sono tutti piani, i primi quattro a rima alternata, e il quinto e il sesto a rima baciata.

L'Assunzione di Dun Karm ha diciotto strofe pindariche e possiede tutte le maggiori qualità tecniche che s'incontrano nell'inno manzoniano, particolarmente la rapidità dell'espressione, le interrogazioni retoriche, il tono sentenzioso che rievoca detti biblici o evangelici. La somiglianza del motivo religioso, svolgendosi secondo una esigenza di celebrare quasi epicamente un contenuto cristiano, ha altresì suggerito una veste esteriore identica, come potrebbe confermare un confronto delle scelte delle rime che legano anche qui ogni strofa con la seguente. La suggestione manzoniana non si intravvede soltanto nell'uso dello schema ma anche nella scelta degli stessi suoni tronchi, specialmente in chiusura: camminò - posò; risalì - ordì; fral-risal; baciò - rallegrò; vestir-morir; odor-splendor; cadè-piè; cammin-cittadin; lassù-quaggiù.

(1) Cfr., ad esempio, Per novello sacerdote - I, Il naufragio di San Paolo a Malta, Prima Comunione. (2) Cfr., fra l'altro, A Leopoldo Dagradi e A Cristoforo Colombo. 
Ci sono anche aspetti della sceneggiatura del quadro biblico che avvicinano di più le due opere. Il Manzoni anima così il brano evangelico:

Tale il marmo inoperoso

che premea l'arca scavata,

gittò via quel Vigoroso (3).

Dun Karm sceglie le stesse rime, usa un verbo incisivo che riecheggia gittò, che è, anzi, quasi un suo sinonimo, e parla di Cristo attraverso la stessa antonomasia:

Ma dal grembo tenebroso

della vita che l'accolse,

balzò via quel Vigoroso (4).

La dodicesima quartina di Il-Ghid il-Kbir, scritta con senari doppi, un metro spesso adoperato nei tempi moderni dal Prati e dal Bertacchi, fra tanti altri, e scelto dal Manzoni stesso per il coro Dagli atrii muscosi, rievoca fedelmente la decima strofa della Risurrezione, dove il poeta si rifà al testo latino del Vangelo di Matteo (5), e traduce drammaticamente il quadro, dando il massimo rilievo all'identità vaga e lucente dell'angelo e alla brevità incisiva delle sue parole:

Un estranio giovinetto

si posò sul monumento;

era folgore l'aspetto,

era neve il vestimento;

alla mesta che ' 1 richiese

diè risposta quel cortese:

E risorto; non è qui (6).

La strofa di Il-Ghid il-Kbir conserva tutte Te componenti della scena, scultorea nella sua solenne immutabilità, e utilizzando lo stesso nucleo lessicale e figurativo, crea un quadro identico nel complesso e nei particolari:

Bil-qieghda fuq l-ghatu, bhal berqa dawl wiccu,

bhas-silg bojod hwejgu raw qieghed zaghzugh,

li b'lehen ta' hlewwa fis qal'lhom: Xejn tibzghu,

mill-mewt qam kif wieghed: naha ohra ssibuh (7).

L'unico dettaglio che manca nel testo manzoniano e che è incluso nel brano maltese sta nelle parole, trovate nel Vangelo, «mill-mewt

(3) Vv. 22-24

(5) " Angelus enim Domini descendit de coelo et accedens revolvit lapidem, et sedebat super eum. Erat autem aspectus eius sicut fulgur: et vestimentum eius sicut nix. Prae timore autem eius exterriti sunt custodes, et facti sunt velut mortui. Respondens autem angelus dixit mulieribus: Nolite timere vos ... Non est hic: surrexit enim, sicut dixit" (Matt., XXVIII, vv. 1-6).

(6) Vv. 64-70

- (7) Vv., 41-44. Il verso 67 della Risurrezione, citato sopra, è riecheggiato da Dun Karm anche nel seguente: "Era di neve l'ondeggiante veste " (L'Annunziata, v. 3) e in un brano in maltese: Wiccha jiddi / (...) bhaz-zerniq tà jum sábih, / dawl ix-xemx il-libsa taghha (Innu lil Marija tielgha s-sema, vv. 9-11). 
qam kif wieghed " che il Manzoni, comunque, inserisce nel verso 82 dello stesso inno: « $\dot{\mathrm{E}}$ risorto, come il disse ». Dopo aver completato il quadretto, il Manzoni elabora poeticamente uno dei momenti liturgici della commemorazione pasquale, cioè quando il sacerdote invita i fedeli alla letizia (8). Dun Karm intreccia anche lui questo rituale e rifacendosi al testo manzoniano che ripete due volte la parola godi nei versi 79 e 80, inserisce due volte l'imperativo ifirhu nei versi 47 e 49 . L'ultima parte dell'inno italiano, quella che va dal verso 85 fino alla fine, si scioglie in un invito alla gioia e alla carità per i deboli e in un gentile ammonimento morale. Dun Karm segue il suo maestro anche sotto questo aspetto strutturale e utilizza l'ultima parte del suo inno per un simile avvertimento (9).

Un simile parallelismo si intravvede ancora tra La Pentecoste, ispirata alla discesa dello Spirito Santo sugli apostoli, e Il naufragio di San Paolo a Malta in cui Dun Karm crea fantasticamente una specie di pentecoste speciale, fuori delle vicende evangeliche e posta nella storia nazionale, che si svolge mediante la stessa drammaticità visionaria. Nella prima parte il Manzoni celebra la grandezza della Chiesa trionfante e militante (vv. 1-48) e poi passa a meditare il significato spirituale della vicenda religiosa (vv. 49-50), indicando la trasformazione umana che doveva seguire.

Come ha detto il Tommaseo, l'idea dell'inno è che la Chiesa è la società che fu fondata per rinnovare la terra (10). Dun Karm apre l'inno pindarico con la rievocazione dell'evento biblico, considerato come un momento di maggiore importanza nel processo storico dell'isola (vv. 1-48), e poi passa a riflettere sugli effetti immediati che questa diversa «discesa » divina sul paese era destinata a produrre. Insieme al democratismo e al popolarismo politico-sociale (11), si sente pure la presenza di un rinnovamento interiore e spirituale. Il Manzoni parla dei bamboli, delle donzelle, delle vergini, delle spose e dei giovani, mentre il maltese rievoca la fratellanza tra l'odiato e l'odiator, la redenzione sociale e personale della fanciulla debole, della deserta vedova, e la collaborazione tra il ricco e il povero.

(8) Cfr. vv. 85 sgg.

(8) Crr. vv. 85 sgg.. di San Paolo a Malta che dalla descrizione dell'episodio biblico che racconta l'arrivo dell'apostolo passa all'esortazione religiosa.

(10) Ispirazione $e$ arte, Firenze, 1858 , p. 341.

(11) $\mathrm{E}^{\prime}$ complessivamente applicabile a Dun Karm il giudizio gramsciano sulla religiosità manzoniana, cioè che una tale presentazione della religione è la trasformazione della visione dialettica della realtà in un mito; in altre parole, si ha qui un poeta che si serve delle premesse dogmatiche e dei voli ta in un mito; in altre parole, si ha qui un poeta che si serve delle premesse cologmatiche (cfr. A. Gramsci, Manzoni e gli umili, Letteratura e vita nazionale, Torino, Einaudi 195, pp. $72 \mathrm{sgg}$.). Inoltre, mentre Gramsci vede nel Manzoni cantore del popolo un anticipatore del Verga, si può similmente vedere in Dun Karm l'annunziatore dell'ottica realistica fotograficamente fedele alle esperienze dolorose del popolo, che caratterizzerà la narrativa di Guzè Bonnici (1907-1940), l'autore di Imhabba u mibeghda (1927), Il-qawwa tà l-imhabba (1939) e Helsien (1940), e di Guzè Chetcuti (n. 1914), l'autore di Id-dawl tal-hajja (1958), Imhabba u mewt (1961), L-isqaq (1962), It-tnalja (1964) e Nirien tà mhabba (1967). 
L'interpretazione tommaseana conta anche qui, con la sola differenza che il rievocato evento biblico doveva rinnovare un paese, e non tutta la terra. Il motivo universale è tradotto in un motivo nazionale. L'avvicinamento del divino all'umano, dell'ideale al reale, è comune a tutti e due, così che la sublimità che è una parte integrale della natura del primo è concessa ugualmente al secondo. In fondo, si tratta di una posizione fondamentalmente romantica. L'ideale e il vero, una dialettica che in questo caso si configura nella perfezione immutabile di un momento biblico e nella perfettibilità di una esperienza umana, sono entrambi presenti, fusi insieme per sistemarsi organicamente in una sola realtà.

Quello che potrebbe sembrare teatrale nel Manzoni e troppo drammatico in Dun Karm, cioè la componente fantastica che rielabora un dato di storia ideale, è ridimensionato secondo un vero storico, del tutto umano. Essenzialmente, queste considerazioni sono applicazioni morali della interpretazione moderna del principio d'imitazione della natura, rinnovato dai neoclassici, da un Foscolo e soprattutto da un Winckelmann.

Sia La Pentecoste sia Il naufragio di San Paolo a Malta si legano a coppia dalle rime del verso finale delle strofe, che è sempre tronco. Nell'inno manzoniano il legame che unisce le strofe che vanno dalla terza alla sesta, e poi dall'undicesima alla quattordicesima, è ancora più forte perché si ripete la stessa sillaba rimata: salì-dì; aprì-udì; cor-vincitor; fior-altor. Nell'inno del maltese questo ripetersi, che ha lo scopo di intensificare il legame strofico e di sciogliere il contenuto in chiave musicale, avviene nelle strofe che vanno dalla settima alla decima: odiator-dolor; ognor-vincitor. La scelta delle sillabe rimate manifesta ancora una corrispondenza abbastanza evidente. Inoltre, nelle due opere il primo, il terzo e il quinto verso di ogni ottava sono sdruccioli e liberi di ogni rima, mentre il secondo e il quarto sono piani anch'essi e legati dalla rima baciata.

Un analogo connubio di contenuto e forma è suggerito da un confronto di Il cinque maggio con A Cristoforo Colombo. Le due odi prendono il motivo dal concetto romantico della singolarità individualistica di un personaggio storico, degno di esaltazione e di sopravvivenza storica, ma è un concetto che, al di là dei limiti della visione foscoliana, qui parzialmente presente, si traduce in una prova della grandezza divina che si manifesta attraverso il genio umano. I due poeti tramandano il nome di ciascun personaggio alla posterità, ma è soprattutto la religiosità che anima il processo storico che primeggia. Dal punto di. vista tecnico, Dun Karm segue il carattere dell'ode manzoniana in numerosi elementi esteriori. Ad esempio, le due poesie hanno diciotto 
strofe di sei versi ciascuna, e si assomigliano nel susseguirsi dei versi sdruccioli, piani e tronchi, e nel legame della rima che unisce una strofa alla seguente.

La strofa pindarica, arricchita da una vasta varietà di schemi della rima, trovò grande fortuna negli inni maltesi che il poeta scrisse instancabilmente per un'ampia gamma di motivi, occasioni e personaggi del mondo civile e cristiano. Delle volte, $\mathrm{i}$ suoi inni hanno in realtà la struttura e la sensibilità musicale della canzonetta metastasiana. Di solito la strofa è la quartina, e quando si forma di otto versi, è generalmente riducibile in due quartine, perché il periodo logico e il periodo ritmico suggeriscono una pausa o un tenue distacco tra le due parti (12), come fa, ad esempio, il Mameli in Dio e il popolo.

Seguendo un'altra costante caratteristica dell'innologia italiana, Dun Karm adopera il polimetro per effettuare mutamenti nel tono e per contrastare atteggiamenti diversi di fronte ad un solo argomento (13). Riecheggia pure la tradizione italiana nella scelta dei metri, particolarmente il senario, il settenario, l'ottonario, il decasillabo e l'endecasillabo (14).

Sia in questi inni, in italiano e in maltese, sia in altre opere che prendono lo spunto da un motivo religioso, Dun Karm delinea il personaggio divino secondo i termini figurativi del Manzoni. Ci sono, in primo luogo, le antonomasie. Con l'adoperazione del nome comune invece del proprio, Cristo diventa "il Signore» (15), e «Mulej » (un vocabolo di origine semitica, invece di Sinjur, di derivazione italiana) nelle poesie maltesi (16). Per il Manzoni Dio è il «Re dei re»(17) e il "Re dei regi »(18), ed è « dei regi il sommo Re» per Dun Karm (19), mentre per tutti e due è frequentemente il « $\operatorname{Re} »(20)$, « il Giusto » (21),

(12) Cfr. In-nahal tà Santa Rita, Il-Milted, Via Crucis, Nirien tà mhabba.

(13) Cfr. Innu tà ferh, Innu lì Marija tielgha s-sema, Innu lì San Filippu tà Aggira.

(14) Cfr. Innu lill-Madonna, Innu tà fil-ghaxija, Is-salib u Santa Liena, Via Crucis, Lil Kristu Sultan, Innu tà fil-ghodu, San Guzepp lill-haddiem nisrani, Innu lill-patrijarka, San Guzepp, Rex, Il-ghanja tà Mejju, Innu tal-paggi tà Gesù Sagramentat, Innu lill-qalb tà Gesù, Innu lil San Filippu tà Aggira, Innu tà ferh, Innu lì Marija tielgha s-sema, Nirien tà mhabba, Il-Milied, Lill-qalb imqaddsa tà Gesì.

(15) Manzoni: Il nome di Maria, v. 67; La Passione, vv. 28, 84; La Risurrezione, vv. 14, 31, 32, 111, 112; La Pentecoste, v. 70; Strofe per una prima Comunione, vv. 16, 34, 36; Adelchi, IV, I, vv. 15, 57, 96, e V, VIII, v. 38; Dun Karm: Áve, Signore, vv. 6, 12, 18; Preghiera notturna, v. 20; La' natività di Maria. vv. 24, 41;Corpus Domini, v, 27; Nel giubileo episcopale di Leone XIII, v. 122; A San Filippo d'Aggira, vv. 16, 24, 90; Carità, vv. 1, 18, 27; Per i Congressi Eucaristici, v. 34; A padre Charles Plater S. J., v. 59; Il mare, vv. 42, 51, 83; Prima Comunione, v. 33; Capodanno, vv. 37, 50, 64; A Cristoforo Colombo, v. 94; La predicazione evangelica, vv. 2, 29; La dignità episcopale, v. 42.

(16) Alla, vv. 37, 46; Zjara lì Gesù, v. 120; Innu tà fil-ghodu, vv. 1, 9, 21, 25, 33; Innu tà fil-ghaxija, vv. 1, 13, 25; Pater, vv. 1, 7; Hajr, vv. 1, 5, 9; Imhabba siekta v. 1; Innu tà ferh, v. 22;Innu tax-Xirka tà l-isem imqaddes t'Alla, vv. 1, 25; Lill-pjacir, v. 5; Zjara lil Gesì Sagramentat, v. 1; Talba ghax-xita, vv. 1, 4, 9, 12; Lill-Mons. Dun Vincenz Schembri, v. 8.

(17) Adelchi, V, X, v. 110

(18) Ibid., IV, 'I, v. 100

(19) Per novello sacerdote - I, v. 16; Lil Kristu Sultan, v. 19

(20) Manzoni: Il Natale, vv. 98, 112; La Pentecoste, v, 13; Strofe per una prima Comunione, vv, 27, 43; Dun Karm: A Malta, dopo il Congresso Eucaristico vv. 5, 15; Per i Congressi Eucaristici, v. 36; Lil Kristu Sultan, vv. 2, 28, 34; Il-Monument, v. 34; Innu tal-paggi tà Gesì Sagramentat, vv. 6, 14, 22, 30, 38; Innut lil Gesù, vv. 9, 21; Cristo, v. 3; Il Natale, v. 79; L'Assunzione, v. 108. In altri casi c'è pure un riecheggiamento petrarchesco nell'uso dell'aggettivo superno; il Petrarca scrive « il superno regno " $(A M a r i a$ Vergine, v. 39) e così anche Dun Karm ( $I l$ Natale, v, 4). Una variazione è "il Re superno " (A Cristoforo Colombo, v. 88; Per novello sacerdote - VII v, 3).

(21) Manzoni: La Passione, vv. 25, 27; Dún Karm: Il Natale, v. 10; Mestizia e timore, v. 65. 
« l'Eterno » (22), «il Santo » (23), «il Grande » (24), « il Giudice » (25) e « il Vigoroso » (26).

Un semplice spoglio delle poesie religiose, a cui ci riferiamo, rivela immediatamente che il linguaggio poetico di Dun Karm gira spesso nei limiti del nucleo lessicale fondamentale del Manzoni. Ecco alcuni dei vocaboli più frequenti: nomi: inferno, onor, empiro, grazia, terra, gloria, cielo, regno, sole, tenebre, vittoria, padre, spirito, figlio; aggettivi: eterno, umile, superbo, beato, celeste, santo, divino, superno, immortale, misera; verbi: risonare, rapire discendere, spargere, implorare, sperare. Sono particolarmente care a Dum Karm alcune espressioni figurative o retoriche che fanno parte del frasario elementare o almeno più tipico della creazione manzoniana, e che occorrono ripetute letteralmente o parzialmente modificate in opere sia in italiano sia in maltese, ad esernpio: «fanciulla ebrea » (27), "progenie d'Israello» (28), « disonor del Golgota » (29), "tristo esiglio » (30), « Dio nascosto » (31), «gran Dio» (32).

Non è soltanto la figura guerriera e militante di Cristo che ispira i poeti a drammatizzare il Vangelo, ma è anche la Madonna, quella che il Manzoni chiama "Tuttasanta »(33) e Dun Karm "Tuttapura » (34). Rievocando un detto biblico che delinea scultoreamente l'imma-

(22) Manzoni: La Passione, v, 18. Il Natale v, 44; Adelchi, IV I Coro, vv. 15, 87; Dun Karm: Per la prima messa del sac. G. Gauci, v. 7 ; A San Filippo d'Aggira, v. 15; A Maria Immacolata, v. 22; Il naufragio di San Paolo a Malta, v. 23; Il mare, v. 20. Il maltese riecheggia il brano "Qual ti può dir dei secoli / tu cominciasti meco? " (Il Natale, vv. 45-46) in "Non erano i secoli, / e Tu eri, o Divino " (Il Natale, vv. 63-64). In altri luoghi Dio è descritto come il "Signor di tutti i secoli " (Prima Comunione, v. 33), il "Signor dei secoli " (Per il monumento di Cristo Redentore, v. 29) e il "Rege dei secoli " (Il Natale, v. 79). Cfr. anche Innu tax-Xirka tà l'isem imqaddes t'Alla, vv. 13-16.

(23) Manzoni: La Pentecoste, v. 63; La Passione, v. 29; Strofe per unä prima Comunione, v. 13; Adelchi, I, V, v. 320; Il Natale, v. 24; Dun Karm: Per novello sacerdote - I, v. 127.

(24) Manzoni: Strofe per una prima Comunione, v. 13; Dun Karm: Il Natale, v. 13. Una variazione di questa antonomasia è "il Forte »: Alla Croce, v. 1; Per il monumento di Cristo Redentore, v. 31; It Natale, v. 11; Alla, vv. 3, 4

(25) Manzoni: Strofe per una prima Comunione, v. 43; Dun Karm: Mestizia e timore, v. 65.

(26) Manzoni: La Risurrezione, v. 24; Dun Karm: L'Assunzione, v. 17.

(27) Manzoni: Il nome di Maria, v. 68; Dun Karm: L'Annunziata, v. 6; Bjuda, v. 13.

(28) Cosi scrive Dun Karm in L'Assunzione, v. 4, rifacendosi al v. 69 di Il nome di Maria: "O prole d'Israello ". Cfr. anche "bint Jesse" (Angelus, v. 26) e "tfajla tà Jesse " (Innu tà ferh, v. 5).

(29) Il cinque maggio, v. 101. In una sua lettera il Manzoni parla del significato e dell'origine biblica della frase: "Il 'disonor del Golgota' è imitato dall' ' improperium Christi ' e dall'altro ' stultitiam Crucis ' di San Paolo; i grandi predicatori francesi gettano più d'una volta nei loro discorsi l' 'opprobre de la croix ', senz'altro temperamento, perché s'intenda che è disonore, obbrobrio, improperio agli occhi del mondo " (A Giovan Battista Pagani, 15/11/1821, Opere, VII, Tomo I, a cura di C. Arieti, Verona, A. Mondadori 1970, p. 253). Le citazioni bibliche sono dalla lettera agli Ebrei, XI, v. 26, e dalla prima lettera ai Corinti, I, vv. 18 e 29 rispettivamente. La irase e testualmente riprodotta da Dun Karm in Alla Croce, v. 59. Sono numerose le variazioni ispirate ad essa: "nefando Golgota " (La Chiesa e Leone XIII, v. 7) "l'irto Golgota" (Mestizia e timore, v. 67), "l'empio Golgota " (Per il Congresso Eucaristico internazionale di Malta, v. 180), "il-Kalvarju tal-hatjin " (Dan min hu?, v. 11), "il-Kalvarju bis-sahta " (It Ghid il-Kbir, v. 3). I seguenti brani sviluppano ancora il significato del "disonor ": "E a Lui, che sovra il Golgota / spirava maledetto / reso di scherno e d'odio / vituperato oggetto " (Per novello sacerdote - II, vv. 81-84), "Fuq il-Kalvarju wahxi / ghodda tà mewt mishuta / deh'r is-Salib (Is-Salib u Santa Liena, vv. 1-3), / Ghuda bla gieh, mghobbija / bis-sahta bit-tmagdir » (Is-Salib, vv, 1-2).

(30) Manzoni: Il cinque maggio, v. 46; La Passione, v. 94; Dun Karm: Prima Comunione, v. 39.

(31) Manzoni: Strofe per una prima Comunione, v. 26; Dun Karm: Inno Eucaristico, v. 27; Innu Ewkaristiku, v. 27.

(32) Manzoni: Strofe per una prima Comunione, v. 52; Dun Karm: Alla, v. 1; Innu lil San Filippu tà Aggira, v. 5; Il-Milied, vv. 5, 21; Innu tax-Xirka tà l'isem imqaddes t'Alla, v. 7; Innu Malti, v. 4. In queste poesie maltesi l'autore scrive "kbir Alla", ritenendo l'ordine sintagmatico italiano, invece di "Alla kbir" come esige l'uso normale della lingua.

(33) Il nome di Maria, v. 37.

(34) A Maria Immacolata, v. 73. 
gine di una vergine potente e vittoriosa (35), il Manzoni scrive: inclita come il sol, terribil come

oste scherata in campo (36),

e Dun Karm, ritenendo la stessa figurazione, cambia soltanto qualche vocabolo e riproduce la tonalità del settenario finale dell'inno:

questa è la donna forte

com'oste armata in guerra (37).

E abbastanza evidente che il motivo centrale del Manzoni è identico a quello di Dun Karm. Ė la celebrazione, orientata secondo uno schema della liturgia cattolica, di un "vero» morale (concepito come una causa del bello) che si trova non nella realtà della materia, ma nella vita intima dello spirito che sta cercando una grazia soprannaturale per conoscere il «vero interiore». E la strada battuta per arrivare a questa interpretazione beatifica della realtà terrena non sta soltanto nel sentimento romantico, continuamente presente nella vivacità della visione, ma pure nella meditazione sull'avvenimento religioso che, una volta contemplato, suggerisce la preghiera e l'invocazione; si ha, così, la triplice struttura degli inni manzoniani e dunkarmiani.

Sono inni che presentano un artista straziato tra $i$ due poli estremi di una dialettica: da un lato lo spettacolo festoso, pieno di colori e di suoni, e dall'altro l'esigenza di un raccoglmento mistico, meditativo. Ma ogni momento è svolto secondo « un gran disegno » aprioristico che sembra umano (38), ma che si traduce complessivamente in una visione fantastica di « un divin disegno" (39).

Dun Karm tende anche lui a strutturare tutto secondo una linearità narrativa, e volendo confessare apertamente la propria fervida fede nel cristianesimo e ridurre le verità religiose ad una serie di quadri drammatici e gravi, spesso lascia i concetti dommatici prevalere sul tessuto delle immagini. Ma sono questi gli elementi con cui cerca di fondere la realtà metafisica con la solidarietà del gruppo umano che soffre, e di dare prova della collaborazione tra una vita morale e la poesia. (Del resto, il concetto della necessità di stabilire un rapporto armonico tra verità religiosa e visioni estetiche è alla base della sua poetica) (40).

(35) «Quae est ista, quae progreditur quasi aurora consurgens, pulchra ut luna, electa ut sol, terribilis ut castrorum acies ordinata?" (Cant. VI, v. 9).

(36) Il nome di Maria, vv. 83-84.

(37) La Natività di Maria, vv. 57-58. Nei vv. 63-64 completa il quadro biblico, includendo il paragone con il sole e con la luna: "Più splendida del sole, / più bella della luna ».

(38) Il cinque maggio, v. 38.

(39) Dun Karm: La giustizia divina, v. 13

(40) Cfr., ad esempio, Foglie d'alloro cit., p. 6 e Il-poezija Maltija fl-iskejjel ahjar minn kull wahda ohra, loc. cit., p. 3. Era il Manzoni stesso, fra tanti altri, che, rielaborando il detto oraziano "omne tulit punctum qui miscuit utile dulci, lectorem delectando, pariterque monendo " (Ars poetica, vv. 343-344), aveva asserito che l'oggetto della poesia è il vero, e l'utile è il suo scopo, creando così una "sorgente aveva asserito che l'oggetto della poesia è il vero, e lutile è il suo scopo, creando cosi una "Sorgente
d'un diletto nobile e durevole " (Lettera al marchese Cesare D'Azeglio, loc. cit., pp. 162-163). L'identificazione del vero, del bello, del morale, che forma la base della poetica manzoniana, è al centro della visione di Dun Karm. 
Come il Manzoni, Dun Karm mescola il valore universale degli avvenimenti biblici (il linguaggio e le immagini richiamano spesso diversi brani della bibbia) con la moralità della vita umana che, presentata nella sua interezza costituita del bene e del male, deve necessariamente trovare un posto nel processo della storia terrena.

Il Manzoni degli inni e delle odi, come anche quello delle tragedie e del romanzo (41), si rivolge contemporaneamente al cielo e alla terra con lo scopo di misticizzare la storia e di umanizzare i personaggi religiosi. Dun Karm degli inni, partecipando profondamente alla stessa interpretazione politico-religiosa, e in effetti dando evidenza a quella che il Tommaseo stesso definisce «la bellezza, eziandio, umana del Cristianesimo » (42), arriverà poi anche a dare una sola identità alla religione e alla storia patria, intravvedendo nell'una la motivazione dell'altra, e interpretando la dura lotta nazionale come una conferma dell'attiva presenza di un Dio che si è manifestato ad un popolo attraverso le mutevoli condizioni di tempo e di luogo.

(41) A proposito del capolavoro manzoniano, è opportuno ricordare qui la rassomiglianza tra il paragrafo finale dei Promessi sposi ( La quale, se non v'è dispiaciuta affatto, vogliatene bene a chi l'ha scritta; e anche un pochino a chi l'ha raccomandata. Ma se invece fossimo riusciti ad annoiarvi, credete che non s'è fatto apposta ") e la chiusura di una introduzione che Dun Karm scrisse ad una sua raccolta poetica: «Issa jekk il-ktieb ghogbok u jaghmillek il-gid, aghti tifhir lil Alla li jingeda b'kollox ghall-ahjar tal-bniedem; jekk imbaghad ma joghgbokx, jew ma jiswielek xejn, issabbar, ghaliex ma kellix fehma ndejqek 》 (Dahla, L-ewwel ward jew gemgha tal-poezijiet li ktibt bil-Malti cit., p. 6).

(42) N. Tommaseo - G. Capponi, Carteggio inedito dal 1833 al 1874, I, a cura di I. Del Lungo e P. Prunas, Bologna, Zanichelli 1911-1920, p. 511. 
Arti Grafiche Boccia s.r.l. - Salerno 
son

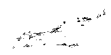


. 